\title{
The accelerator after next
}

\author{
from D. J. Miller
}

\begin{abstract}
"WHY have a conference about the accelerator after next, when we are just building the next one?" That is the question a lot of us were asking as we flew to Hamburg on 22 February for a 6-day working meeting on electronpositron storage rings of $100 \mathrm{GeV}$ (the LEP, Large Electron Positron project). The organisers, ECFA - the European Committee for Future Acceleratorsseemed to be looking a very long way into the future but a number of the participants argued that we should commit ourselves to building such a machine
\end{abstract} as soon as the money can be found.

The reason for their urgency lies in the present theoretical situation. Results from the present $\mathrm{e}^{+} \mathrm{e}^{-}$storage rings, from CERN and from Fermilab are giving increasingly firm and consistent support to the idea of charm and its relation to the neutral current in weak interactions. As a consequence, theorists are building more and more detailed models of elementary particles, and predicting quite specific properties for the forces which govern their interactions. The final unification of the weak, electromagnetic and strong forces has not been written down yet, but many parts of the unification scheme exist and will probably survive in something like their present form. In particular, a family of massive particles, the intermediate vector bosons $\left(\mathrm{W}^{ \pm}\right.$and $\mathrm{Z}^{0}$ ), is required to account for the weakness of the weak interaction, and another family of 'Higgs bosons' is needed to relate the $\mathrm{W}^{ \pm}$ and $\mathrm{Z}^{0}$ to the photon the carrier of the electromagnetic force. Precise predictions exist for the masses of the $\mathrm{W}^{ \pm}$ and $Z^{0}$, though nobody claims that these predictions are unique. It is also expected that, as $\mathrm{e}^{+} \mathrm{e}^{-}$collision energies are increased, the weak interaction should grow in strength compared with the electromagnetic force. At the predicted mass of the $\mathrm{Z}^{0}, 80 \mathrm{GeV} / \mathrm{c}^{2}$ equivalent to $40 \mathrm{GeV}$ on $40 \mathrm{GeV} \mathrm{e} \mathrm{e}^{-}$ collisions - one of three things should happen. There could be a resonance, similar in some ways to the $\psi$ and $\psi^{\prime}$ but even more interesting to analyse because its decays would tell us exactly how the weak neutral current interaction couples to every other kind of particle. There could be a steady increase of the weak interaction rate which would completely swamp the falling electromagnetic rate. Or there might be a slow levelling-off of the increase in the weak interaction rate, with no resonance. Such a result would set a lot of puzzles, but their answer would come directly from a study of the events seen.

If the $\mathrm{Z}^{0}$ resonance is seen, then it is expected that pair production of $\mathrm{W}^{+}$ and $\mathrm{W}^{-}$would also occur at a slightly higher energy, and at these energies it might also be possible to observe the production of Higgs bosons by way of $\mathrm{W}^{ \pm}$or $\mathrm{Z}^{0}$ intermediate states.

Some theorists are sufficiently confident in these predictions that they say we should go ahead as fast as possible and build the LEP machine. Others are more cautious. The first two $15 \mathrm{GeV}$ on $15 \mathrm{GeV} \mathrm{e}^{+} \mathrm{e}^{-}$machines will not work until 1978 (PETRA in Hamburg) or 1979 (PEP in Stanford, California). The CERN SPS is just taking its first data. A large number of the predicted effects will begin to be seen at the 5 to $10 \%$ level with these devices. Perhaps we can get some more indirect evidence about the masses of the $\mathrm{W}^{ \pm}$and $\mathrm{Z}^{0}$ from neutrino interactions. Then there are relatively cheap advances which could be made by adding an antiprotonproton facility $(270 \mathrm{GeV}$ on $270 \mathrm{GeV})$ to the SPS, or by adding a $25 \mathrm{GeV}$ electron ring to collide with $270 \mathrm{GeV}$ SPS protons. Above all, money is tight and may be getting tighter. Who would pay for a new machine costing over one thousand million Swiss francs to build, and consuming over one hundred megawatts of power?

Electron machines are very greedy for power because the beams radiate energy away in the form of synchrotron radiation. That is why it is much easier to build high energy proton accelerators than electron accelerators. But electronpositron collisions seem to be a much better way of doing high energy physics. When protons collide at high energy the really interesting part of the interaction is between the constituent parts of each proton, the three quarks. This means that, on average, only one third of the energy of each beam is actually available in the basic quark-quark collision, compared with $\mathrm{e}^{+} \mathrm{e}^{-}$collisions where all the energy of each beam is concentrated in one particle. In addition, in protonproton interactions, the quark-quark collision is accompanied by a splash of relatively uninteresting particles from the other two 'spectator' quarks in each proton. The most important class of electron-positron collisions is much simpler to understand because the initial particles are completely annihilated, and the final state is produced in a welldefined way.

The ECFA working-meeting reviewed the theoretical and technical case for building an LEP machine. Possible detectors were discussed and it was clear that the cost of detectors for collidingbeam machines goes up with energy in a much gentler way than the cost of detectors for fixed-target machines such as the SPS. Perhaps the most vital discussions were on the feasibility and costs of the $\mathrm{e}^{+} \mathrm{e}^{-}$machine itself. Burt Richter from Stanford had made a preliminary investigation of the parameters of a $100 \mathrm{GeV}$ on $100 \mathrm{GeV}$ storage ring. He based his cost estimates on the PEP ring, but suggested that a much bigger machine might gain a considerable benefit from mass-production of components. Other accelerator designers checked his calculations and confirmed that the machine was certainly feasible, even without great advances in technology. One very desirable technical innovation was pointed out. Large numbers of radio-frequency cavity resonators are needed all round the machine to provide the accelerating field which replaces beam energy lost in synchrotron radiation. But half the energy supplied to these cavities does not go into the beam; it is dissipated by resistive losses in the cavity walls. If reliable superconducting cavities can be developed these losses could be almost eliminated. Small laboratory models have been built, but a major programme of research will be needed before production models of superconducting cavities can be designed.

The final opinion of the meeting was very positive. This is the next major accelerator project that should be proposed. It is probably not too big for Europe to build on her own, although collaboration from the USA or elsewhere might be desirable. It would give direct answers to the most important theoretical questions, whereas all other machines, existing or planned, will only give indirect answers. What must now be decided is how to match an LEP project to existing commitments and how to convince governments that the expenses will be worthwhile. 